\title{
Genetic Analysis of Calving Difficulty and Stillbirth in Norwegian Red Cows
}

\author{
B. Heringstad, ${ }^{*}{ }^{1}$ Y. M. Chang, $\neq$ M. Svendsen, $\dagger$ and D. Gianola* $\S$ \\ *Department of Animal and Aquacultural Sciences, Norwegian University of Life Sciences, PO Box 5003, N-1432 Ås, Norway \\ †Geno Breeding and A. I. Association, PO Box 5003, N-1432 Ås, Norway \\ ‡Division of Genetic Epidemiology, Cancer Research UK Clinical Centre, St James's University Hospital, Leeds, LS9 7TF, United Kingdom \\ $\S$ Department of Dairy Science, University of Wisconsin, Madison 53706
}

\begin{abstract}
The objectives of this study were to infer genetic parameters for stillbirth (SB) and calving difficulty (CD) and to evaluate phenotypic and genetic change for these traits in the Norwegian Red breed. Stillbirth is recorded as a binary trait and calving difficulty has 3 categories: 1) easy calving, 2) slight problems, and 3) difficult calving. The overall mean frequency of SB in Norwegian Red was $3 \%$ at first calving and $1.5 \%$ for second and later calvings; mean frequency of the category "difficult calving" was 2 to $3 \%$ for heifers and $1 \%$ for cows at second and later calvings. Mean stillbirth rate has remained unchanged from 1978 to 2004. The proportion of the category "difficult calving" has not changed over the years, but the "slight problems" category increased from 4 to $7 \%$ for heifers and from 2 to $3 \%$ for cows. A total of 528,475 first-calving records were analyzed with a Bayesian bivariate sire-maternal grandsire threshold liability model. Posterior means of direct and maternal heritabilities were 0.13 and 0.09 for $\mathrm{CD}$, and 0.07 and 0.08 for SB, respectively. Strong genetic correlations were found between direct SB and direct CD (0.79), and between maternal SB and maternal CD (0.62), whereas all genetic correlations between direct and maternal effects within or between traits were close to zero. These positive correlations are favorable in the sense that selection for one of the traits would result in a favorable selection response for the second trait. No genetic correlations between direct and maternal effects imply that bulls should be evaluated both as sire of the calf (direct) and sire of the cow (maternal). No genetic change for SB was found, and a slight genetic improvement for CD was detected.
\end{abstract}

Key words: calving difficulty, genetic parameter, stillbirth, threshold model

Received November 28, 2006.

Accepted March 26, 2007.

${ }^{1}$ Corresponding author: bjorg.heringstad@umb.no

\section{INTRODUCTION}

Stillbirth and calving difficulty are important functional traits in dairy cattle breeding, from both economic and animal welfare points of view. Increasing stillbirth rates and undesirable genetic trends for stillbirth have been reported for Holsteins in Denmark (Hansen et al., 2004c) and Sweden (Steinbock et al., 2003). For primiparous Holstein cows in United States, the incidence of stillbirth increased from $9.5 \%$ in 1985 to $13.2 \%$ in 1996 (Meyer et al., 2001a). Stillbirth rates of 10 to $13 \%$ at first calving in Holsteins is of international concern (Philipsson et al., 2006) and has led to an increased interest in calving traits for dairy cattle breeding.

Many studies of genetic parameters of stillbirth and calving difficulty have applied linear models (e.g., Luo et al., 1999; Meyer et al., 2001b; Jamrozik et al., 2005), or single-trait threshold models (e.g., Steinbock et al., 2003; Wiggans et al., 2003; Hansen et al., 2004a,b). Gevrekçi et al. (2006) analyzed stillbirth and calving difficulty in multiparous American Holstein cows with a bivariate sire-maternal grandsire threshold model. These authors found direct and maternal heritabilities between 0.04 and 0.07; genetic correlations were 0.77 between direct effects, 0.84 between maternal effects, and close to zero between direct and maternal effects within and across traits.

Calving difficulty and stillbirth have been a part of the total merit index used for selection of Norwegian Red sires since 1978 (Geno, 2006). For these traits, bulls are genetically evaluated as sire of the calf (direct effect) and sire of the dam (maternal effect). In 2006, the relative weight placed on each of these traits in the total merit index was $1 \%$ (Geno, 2006). The low relative weight for these traits reflects the low frequencies of stillbirth and calving difficulties in the Norwegian Red population.

The objectives of this study were to infer genetic parameters of calving difficulty and stillbirth using a bivariate sire-maternal grandsire threshold model, and 
to evaluate phenotypic and genetic change for these traits in the Norwegian Red breed.

\section{MATERIALS AND METHODS}

\section{Data}

Data were from the Norwegian Dairy Herd Recording System, where stillbirth (SB) was available for all calving records since 1978. Calving difficulty $($ CD) has been scored routinely for all calving records since 1989 . Before 1989, CD was recorded only for first-lactation daughters of test bulls as part of a special recording scheme for progeny testing. For description of phenotypic trends (all parities), all calving records since 1978 (5.5 million) were used for $\mathrm{SB}$, and all records since 1989 (3.2 million) were used for CD.

For the genetic analyses, a data set with first-calving records from 1989 to 2004 was extracted. We included only records for which both sire of calf and sire of the cow [i.e., maternal grandsire (mgs) of the calf] were known Norwegian Red AI sires. Records from multiple births, abortions, or with unknown sex of calf $(0.7,0.5$, and $0.3 \%$ of the records, respectively) were excluded. The data set was further restricted to records with age at first calving between 21 and $34 \mathrm{mo}$, and from herdyear classes with at least 5 first-lactation cows. The final data set had 528,475 records and a total of 1,548 bulls that were sires, mgs, or both; 85,255 herd-year classes were represented.

Stillbirth was recorded as a binary trait $(0=$ born alive or $1=$ dead at birth or within $24 \mathrm{~h}$ ), whereas CD had 3 categories $(1=$ easy calving, $2=$ slight problems, and $3=$ difficult calving). All calving records had information on SB, and $97.6 \%$ had a CD score. For first calving the overall mean frequency of SB was $2.7 \%$, and a total of $92.3 \%$ of the cows had easy calving, $5.3 \%$ had slight problems, and $2.4 \%$ had a difficult calving.

A sire pedigree file with 2,155 individuals was built by tracing the relationships via sires and mgs of the 1,548 bulls with records in the data set, back to 8 generations. A total of 215 males, of which $99 \%$ were born before 1980, had unknown sire or mgs, and these represent the base population in this study.

\section{Model}

A bivariate sire-mgs threshold liability model (Gianola and Foulley, 1983) was used for analysis of binary SB and of the ordered categorical CD. For each trait, the threshold model postulates an underlying continuous variable, liability $(\lambda)$, such that the observed categorical variable takes value $j$ if $\mathrm{T}_{j-1} \leq \lambda<\mathrm{T}_{j}$, where $\mathrm{T}_{j-1}$ and $\mathrm{T}_{j}$ are thresholds, and $j=1,2, \ldots, \mathrm{J}$ indexes the category to which the observation belongs. The thresholds must satisfy $-\infty=\mathrm{T}_{0} \leq \mathrm{T}_{1} \leq \cdots \leq \mathrm{T}_{\mathrm{J}}=\infty$. The threshold $\mathrm{T}_{1}$ was set to zero for each of the traits, because the parameter cannot be identified in a probit analysis. In matrix notation the model fitted can be written as

$$
\lambda=\mathbf{X} \boldsymbol{\beta}+\mathbf{Z}_{\mathbf{h}} \mathbf{h}+\mathbf{Z}_{\mathbf{u}} \mathbf{u}+\mathbf{e}
$$

where $\lambda$ is a vector of unobserved liabilities to CD and $\mathrm{SB} ; \boldsymbol{\beta}$ is a vector of trait-specific systematic effects, including sex of calf ( 2 levels), age at first calving in months (14 levels), and month-year of first calving (168 levels) effects; $\mathbf{h}$ is a vector of herd-year of calving effects $(85,255$ levels $) ; \mathbf{u}^{\prime}=\left[\mathbf{u}_{C D}^{\prime} \mathbf{u}_{S B}^{\prime}\right]$ is a vector of effects of the sire (s) and mgs of the calf for CD and SB, with $\mathbf{u}_{C D}^{\prime}=$ $\left[\mathbf{s}_{C D}^{\prime} \mathbf{m g s} \mathbf{s}_{C D}^{\prime}\right]$ and $\mathbf{u}_{S B}^{\prime}=\left[\mathbf{s}_{S B}^{\prime} \mathbf{m g s} \mathbf{s}_{S B}^{\prime}\right] ; \mathbf{e}$ is a vector of residuals, and $\mathbf{X}, \mathbf{Z}_{\mathbf{h}}$, and $\mathbf{Z}_{\mathbf{u}}$ are the corresponding incidence matrices. Sire and mgs effects were assumed to be correlated, and to follow the multivariate normal distribution, $\mathbf{u} \sim N\left(\mathbf{0}, \mathbf{G}_{\mathbf{0}} \otimes \mathbf{A}\right)$, where

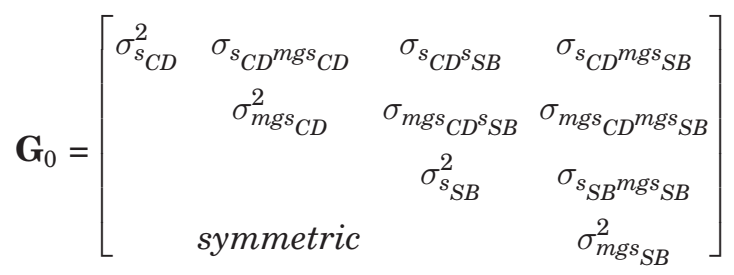

is the sire-mgs covariance matrix for the 2 traits and $\mathbf{A}$ is the matrix of additive genetic relationships among bulls. Effects of different herd-years were assumed to be normally and independently distributed:

$$
\mathbf{h} \sim N\left(\mathbf{0}, \mathbf{H}_{\mathbf{0}} \otimes \mathbf{I}\right),
$$

where $\mathbf{H}_{0}=\left[\begin{array}{cc}\sigma_{h_{C D}}^{2} & \sigma_{h_{C D} h_{S B}} \\ \sigma_{h_{C D} h_{S B}} & \sigma_{h_{S B}}^{2}\end{array}\right]$ is the (co)variance matrix and $\mathbf{I}$ is an identity matrix. Residuals were assumed to follow the normal distribution

$$
\mathbf{e} \sim N\left(\mathbf{0}, \mathbf{R}_{\mathbf{0}} \otimes \mathbf{I}\right)
$$

where $\mathbf{R}_{0}=\left[\begin{array}{cc}1 & \sigma_{e_{C D}{ }^{e} S B} \\ \sigma_{e_{C D}{ }^{e} S B} & 1\end{array}\right]$ is the residual (co)variance matrix. The residual variances for the liabilities of each of the categorical traits were set equal to 1 to attain parameter identification. A Bayesian approach using Markov chain Monte Carlo methods (e.g., Sorensen and Gianola, 2002) was implemented.

Prior Distributions. Independent uniform priors were assigned to each of the elements of $\boldsymbol{\beta}$. As stated above, independent multivariate normal prior distribu- 
tions were used for herd-year (h) and sire-mgs (u) effects, whereas independent inverse Wishart prior distributions were assigned to $\mathbf{H}_{\mathbf{0}}$ and $\mathbf{G}_{\mathbf{0}}$, the (co)variance matrices of $\mathbf{h}$ and $\mathbf{u}$, respectively. The residual covariance (or correlation), $\sigma_{e 1 e 2}$, was assigned a uniform bounded $(-1,1)$ prior. The second threshold $\left(\mathrm{T}_{2}\right)$ for $\mathrm{CD}$ was assumed to be distributed, a priori, as an ordered statistic from a uniform distribution, where $0<\mathrm{T}_{2}$.

Sampling and Convergence Diagnostics. Draws from the posterior distributions of the parameters, except for the second threshold for CD and the residual correlation, were obtained using a Gibbs sampler (Sorensen and Gianola, 2002). For cows that did not have data for CD, "missing liabilities" were included in the augmented posterior distribution. The method of Albert and Chib (1997) was used for sampling the threshold, using a Metropolis algorithm, as described by Chang et al. (2006). Based on trace plots and the convergence diagnostics method of Raftery and Lewis (1992), a chain with a total length of 200,000 iterations was run. The first 10,000 samples were discarded as burn-in and all remaining 190,000 samples were kept for posterior inferences. The effective sample size for the parameters was smallest for herd variance for SB $(1,310)$, and varied between 5,429 and 12,667 for heritabilities and genetic correlations. Acceptance rates for the Metropolis algorithms were $41 \%$ for the threshold for CD and $25 \%$ for the residual correlation.

Direct and Maternal (Co)variances. Sire $\left(\sigma_{s}^{2}\right)$ and mgs $\left(\sigma_{m g s}^{2}\right)$ variances and covariance $\left(\sigma_{s, m g s}^{2}\right)$ for each trait were converted to direct (D) and maternal (M) genetic (co)variances using the relationship

$$
\left[\begin{array}{c}
\sigma_{D}^{2} \\
\sigma_{D M} \\
\sigma_{M}^{2}
\end{array}\right]=\left[\begin{array}{ccc}
4 & 0 & 0 \\
-2 & 4 & 0 \\
1 & -4 & 4
\end{array}\right]\left[\begin{array}{c}
\sigma_{s}^{2} \\
\sigma_{s, m g s} \\
\sigma_{m g s}^{2}
\end{array}\right]
$$

where $\sigma_{D}^{2}$ and $\sigma_{M}^{2}$ are direct and maternal additive genetic variances, respectively, and $\sigma_{D M}$ is the additive genetic covariance between direct and maternal effects (Wiggans et al., 2003).

The phenotypic variance was $\sigma_{p}^{2}=\sigma_{s}^{2}+2 \sigma_{s, m g s}+$ $\sigma_{m g s}^{2}+\sigma_{e}^{2}$ and direct and maternal heritability were calculated as $h_{D}^{2}=\sigma_{D}^{2} / \sigma_{p}^{2}$ and $h_{M}^{2}=\sigma_{M}^{2} / \sigma_{p}^{2}$, respectively.

Additive genetic covariances between direct and maternal effects between SB and CD were obtained using (Luo et al., 1999):

$$
\left[\begin{array}{c}
\sigma_{D_{S B} D_{C D}} \\
\sigma_{D_{S B} M_{C D}} \\
\sigma_{M_{S B} D_{C D}} \\
\sigma_{M_{S B} M_{C D}}
\end{array}\right]=\left[\begin{array}{cccc}
4 & 0 & 0 & 0 \\
-2 & 4 & 0 & 0 \\
-2 & 0 & 4 & 0 \\
1 & -2 & -2 & 4
\end{array}\right]\left[\begin{array}{c}
\sigma_{s_{S B}^{s} C D} \\
\sigma_{s_{S B} m g s_{C D}} \\
\sigma_{m g^{s}}{ }^{s}{ }^{s} D \\
\sigma_{m s_{S B}}{ }^{m g s} C D
\end{array}\right] .
$$

For each sample, a check was made that the $4 \times 4$ covariance matrix of direct and maternal additive genetic effects for the 2 traits was positive definite. All samples had positive determinants.

\section{Norwegian Red}

More than $95 \%$ of the dairy cows in Norway are Norwegian Red, and the population consists of approximately 280,000 cows, of which $98 \%$ participate in the Norwegian Dairy Herd Recording System. All herds participating in the recording system are active in the breeding program, and the best cows in these herds are elite-cows and potential bull-dams. Each year approximately 330 bull-calves from elite sires and elite dams are selected for performance testing. Around 130 of them are selected as test sires, and are progeny tested based on 250 to 300 daughters each. Of these, 10 to 12 are selected as elite sires based on their total merit index and average relationship with the population. About $90 \%$ of calves born are sired by Norwegian Red AI sires, $40 \%$ by test sires, and $60 \%$ by elite sires.

\section{Genetic Evaluation of Sires and Genetic Change}

Bulls were evaluated both as sire of calf (direct effect) and as sire of the cow [i.e., mgs of the calf (maternal effect)]. Sire posterior mean (liability scale) was used as genetic evaluation for direct effect, whereas genetic evaluation for maternal effect for sire $i\left(m a t_{i}\right)$ was calculated as $m a t_{i}=m \bar{g} s_{i}-0.5 \bar{s}_{i}$ where $m \bar{g} s_{i}$ is the posterior mean of mgs effect, and $\bar{s}_{i}$ is the sire posterior mean for sire $i$.

Genetic change for direct and maternal effects on the 2 traits was assessed by plotting average sire posterior means against birth year of progeny (direct effect), and average genetic evaluation of maternal effect ( $m a t$ ) by year of calving of daughters. All calving records of the 1,548 sires and mgs were included (about 900,000 firstcalving records) for calculation of genetic change. Thus, sires were weighted according to their number of progeny to reflect possible genetic change in the Norwegian Red population.

\section{RESULTS AND DISCUSSION}

\section{Phenotypic Level and Trends}

The phenotypic trends for stillbirth and calving difficulty for heifers and cows displayed in Figures 1 and 2 indicate low levels of these problems in the Norwegian Red population. The mean frequency of stillbirth has remained essentially unchanged from 1978 to 2004 (Figure 1), with an overall mean for all parities of about $2 \%$. Stillbirth rate was around $3 \%$ for first calving, and 


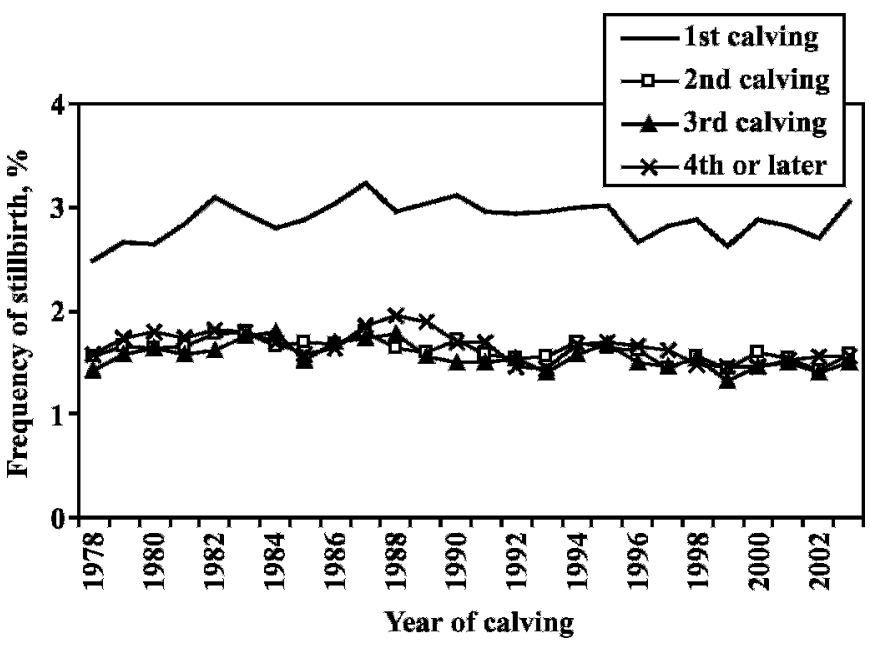

Figure 1. Frequency of stillbirth in Norwegian Red by year of calving for heifers and cows.

$1.5 \%$ for second and later calvings. Mean frequency of the CD categories "slight problems" and "difficult calving" by year are in Figure 2. The frequencies of CD categories were similar for all parities after first calving. Figure 2 shows that between 1991 and 2001 there was a slight increase in the proportion of "slight problems" (from 4 to $7 \%$ for first calving, and from 2 to $3 \%$ for second and later calvings). The frequency of the category "difficult calving" (2 to $3 \%$ for heifers and $1 \%$ for cows) has not changed much over the years.

The stillbirth frequency for Norwegian Red of 3\% for first calving is much lower than for other breeds, and the unchanged level over time is in contrast to other reports. For instance, the stillbirth rate for first-calving Holstein cows in the United States increased from 9.5\% in 1985 to 13.2\% in 1996 (Meyer et al., 2001a). Hansen et al. (2004c) found that the stillbirth frequency for first-calving Danish Holstein increased from 7\% in 1992 to $9 \%$ in 2002. Also, the stillbirth rate for Swedish Holstein heifers has increased over the past 10 to $15 \mathrm{yr}$, and about $10 \%$ of the calves are born dead or die within the first $24 \mathrm{~h}$ (Steinbock et al., 2003). In Swedish Red there has been a slight increase in stillbirth frequency over the last $25 \mathrm{yr}$, from less than 4 to around 5\% for heifers, and from 3 to $4.5 \%$ for Swedish Red cows (Philipsson et al., 2006).

Although CD is more difficult to compare between populations because the scoring systems vary between countries, the frequency of difficult calving in Norwegian Red (overall mean frequency of $1.5 \%$ ) is clearly lower than for other breeds. Hansen et al. (2004c) found that the frequency of difficult calving for first-calving Danish Holsteins decreased from 15\% in 1985 to $9 \%$ in 2002. In Sweden, the mean frequency of difficult calv-
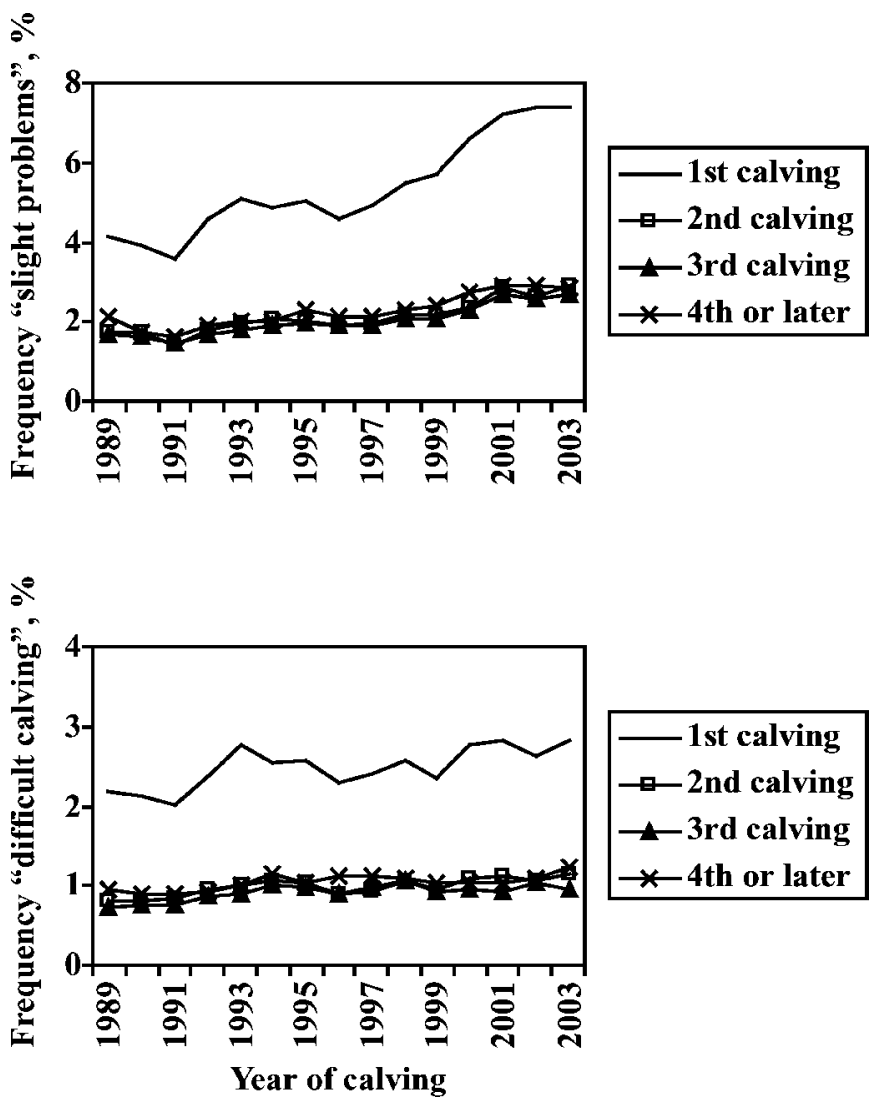

Figure 2. Frequency of the calving difficulty categories "slight problems" and "difficult calving" in Norwegian Red heifers and cows by year of calving.

ings in first parity was $4 \%$ for SRB and $8 \%$ for Holstein (Philipsson et al., 2006). In a study including 404,460 American Holstein cows (all parities), Gevrekçi et al. (2006) reported $73.1 \%$ easy calving, $13.2 \%$ slight problems, and $13.7 \%$ difficult calving (categories included here were "needed assistance" and "considerable force"). In a study of first-lactation Canadian Holstein cows, $19 \%$ of the male calves and $13 \%$ of the female calves were born with difficulty, where the categories were "hard pull" or "surgery needed" (Luo et al., 1999).

The lower frequency of CD and SB in Norwegian Red than in other breeds agrees with Heins et al. (2006), who compared calving difficulty and stillbirth of pure Holsteins vs. crossbreds. In their study, "Scandinavian Red" included both Norwegian Red and Swedish Red. Calves from Holstein first-calf heifers sired by Scandinavian Red bulls had significantly less calving difficulty $(5.5 \%)$ and lower stillbirth rate $(7.7 \%)$ than calves sired by Holstein bulls (16.4\% difficult calving and $15.1 \%$ stillbirth). Heins et al. (2006) also showed that Scandinavian Red/Holstein crossbred dams had a lower frequency of calving difficulty (3.7\%) and stillbirth (5.1\%) 


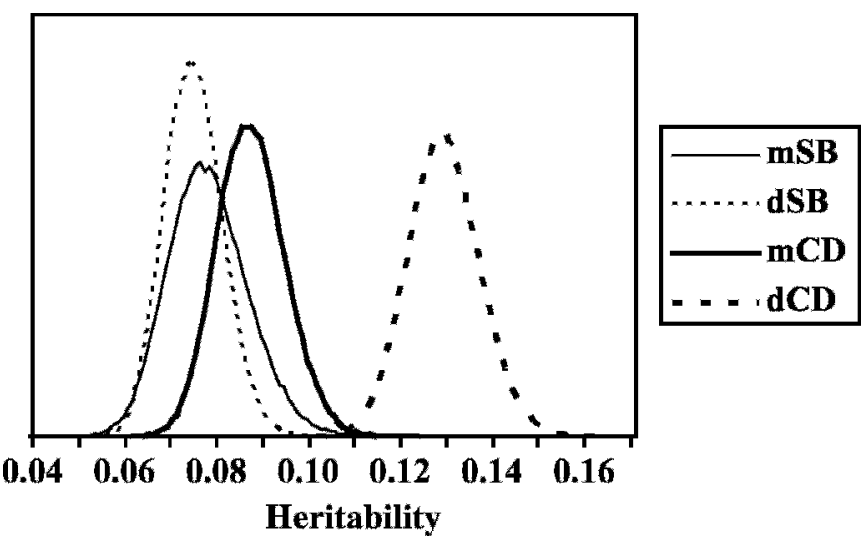

Figure 3. Posterior distributions of direct (d) and maternal (m) heritability of liability to stillbirth (SB) and calving difficulty (CD).

at first calving than pure Holstein heifers (17.7\% difficult calving and $14 \%$ stillbirths).

\section{Heritability}

Posterior distributions of direct and maternal heritability of liability to SB and CD are given in Figure 3. For CD, direct heritability had a higher posterior mean (0.13 vs. 0.09 ) than maternal heritability, whereas for $\mathrm{SB}$, the posterior distributions of direct and maternal heritability were more overlapping, with means of 0.07 and 0.08 , respectively. These point estimates are in agreement with other recent threshold model estimates ranging from 0.04 to 0.12 for direct heritability and from 0.07 to 0.13 for maternal heritability for SB (Steinbock et al., 2003; Hansen et al., 2004b; Gevrekçi et al., 2006). Recent threshold model estimates of heritability for CD or calving ease range from 0.06 to 0.17 for direct heritability and from 0.04 to 0.12 for maternal heritability (Luo et al., 2002; Steinbock et al., 2003; Wiggans et al., 2003; Hansen et al., 2004a; Gevrekçi et al., 2006). Linear model estimates of heritability of SB and CD (e.g., Luo et al., 1999; Meyer et al., 2001b; Jamrozik et al., 2005) in general are lower than those obtained from threshold models.

\section{Correlations}

Posterior distributions of the genetic correlations between direct and maternal effects within trait are given in Figure 4. Posterior mean was close to zero for both SB (-0.02) and CD (-0.03) (Table 1), which is in agreement with Gevrekçi et al. (2006). Hansen et al. (2004a) found similar results for SB in Danish Holstein, but a weak positive correlation between direct and maternal CD, with a posterior mean (SD) of $0.21(0.08)$. Wiggans et al. (2003) reported a genetic correlation

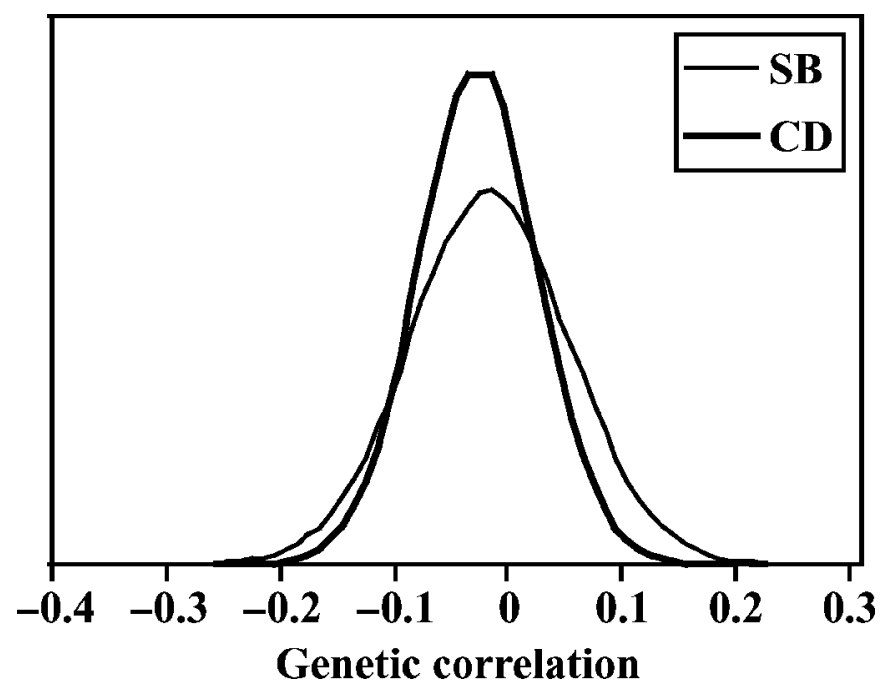

Figure 4. Posterior distributions of genetic correlation between direct and maternal effects for stillbirth (SB) and calving difficulty (CD).

of 0.12 between direct and maternal calving ease in American Holsteins. Some other authors have reported antagonistic genetic relationships between direct and maternal effects. For Canadian Holstein, Luo et al. (1999) found negative correlations between direct and maternal effects for calving ease $(-0.16)$ and for SB $(-0.24)$, and Steinbock et al. (2003) estimated a genetic correlation of -0.10 between direct and maternal components for both SB and CD in Swedish Holstein. The posterior distributions of the genetic correlations between direct and maternal SB and CD are given in Figure 5. Strong positive genetic correlations between direct $\mathrm{CD}$ and direct SB (0.79) and between maternal CD and maternal SB (0.62; Table 1$)$ were in agreement with previous studies. Recent estimates of genetic correlations between $\mathrm{CD}$ and SB range from 0.77 to 0.83 between direct effects, and from 0.62 to 0.84 between maternal effects (Steinbock et al., 2003; Hansen et al., 2004a; Gevrekçi et al., 2006). Here, positive correlations are favorable in the sense that selection for one of the traits would result in a favorable selection response for the second trait.

Posterior mean (SD) of the genetic correlation between direct $\mathrm{CD}$ and maternal SB and between direct SB and maternal CD were 0.11 (0.06) and $0.06(0.06)$, respectively (Table 1). Other studies have reported estimates ranging from 0.04 to 0.15 for the genetic correlation between direct SB and maternal CD, and from 0.06 to 0.08 for the genetic correlation between maternal SB and direct CD (Luo et al., 1999; Hansen et al., 2004a; Gevrekçi et al., 2006).

The lack of a sizable genetic correlation between direct and maternal effects for SB and $\mathrm{CD}$ indicates that 
Table 1. Mean, SD, and 95\% probability interval (2.5 and 97.5 percentiles) of posterior distributions of direct (d) and maternal (m) genetic, herd-year, and residual (co)variances, threshold, heritabilities, and correlations from the bivariate threshold model analysis of stillbirth (SB) and calving difficulty (CD)

\begin{tabular}{llrrr}
\hline Parameter & \multicolumn{1}{c}{ Trait } & Mean & SD & 95\% probability interval \\
\hline Genetic variance & dSB & 0.079 & 0.007 & $0.066,0.092$ \\
& dCD & 0.142 & 0.009 & $0.125,0.159$ \\
mSB & 0.082 & 0.009 & $0.066,0.092$ \\
Genetic covariance & mCD & 0.096 & 0.008 & $0.080,0.112$ \\
& dSB with mSB & -0.002 & 0.006 & $-0.013,0.009$ \\
& dCD with mCD & -0.004 & 0.006 & $-0.016,0.009$ \\
& dSB with dCD & 0.084 & 0.006 & $0.072,0.097$ \\
& dSB with mCD & 0.005 & 0.005 & $-0.006,0.015$ \\
Herd-year variance & mSB with dCD & 0.012 & 0.007 & $-0.001,0.026$ \\
& mSB with mCD & 0.055 & 0.007 & $0.042,0.068$ \\
Herd-year covariance & SB & 0.113 & 0.006 & $0.102,0.124$ \\
Threshold 2 & CD with CD & 0.333 & 0.006 & $0.321,0.345$ \\
Heritability & CD & 0.127 & 0.005 & $0.118,0.136$ \\
& dSB & 0.653 & 0.004 & $0.645,0.661$ \\
& dCD & 0.07 & 0.006 & $0.06,0.09$ \\
Genetic correlation & mSB & 0.13 & 0.008 & $0.11,0.14$ \\
& mCD & 0.08 & 0.009 & $0.06,0.09$ \\
& dSB with mSB & 0.09 & 0.007 & $0.07,0.10$ \\
& dCD with mCD & -0.02 & 0.070 & $-0.16,0.12$ \\
& dSB with dCD & 0.79 & 0.052 & $-0.13,0.07$ \\
dSB with mCD & 0.06 & 0.026 & $0.74,0.84$ \\
Rerd-year correlation & mSB with dCD & 0.11 & 0.062 & $-0.06,0.18$ \\
\hline & mSB with mCD & 0.62 & 0.063 & $-0.01,0.24$ \\
& SB with CD & 0.66 & 0.021 & $0.53,0.71$ \\
& SB with CD & 0.06 & 0.002 & $0.62,0.70$ \\
& & & & $0.06,0.07$ \\
\hline
\end{tabular}

bulls should be evaluated both as sires and maternal grandsires for these traits. Although large genetic correlations were found between direct effects, as well as between maternal effects, it is evident that SB and CD are not the same trait, and both should be included in the breeding objective for a population.

The correlation between herd-year effects was positive (0.66), whereas the residual correlation between

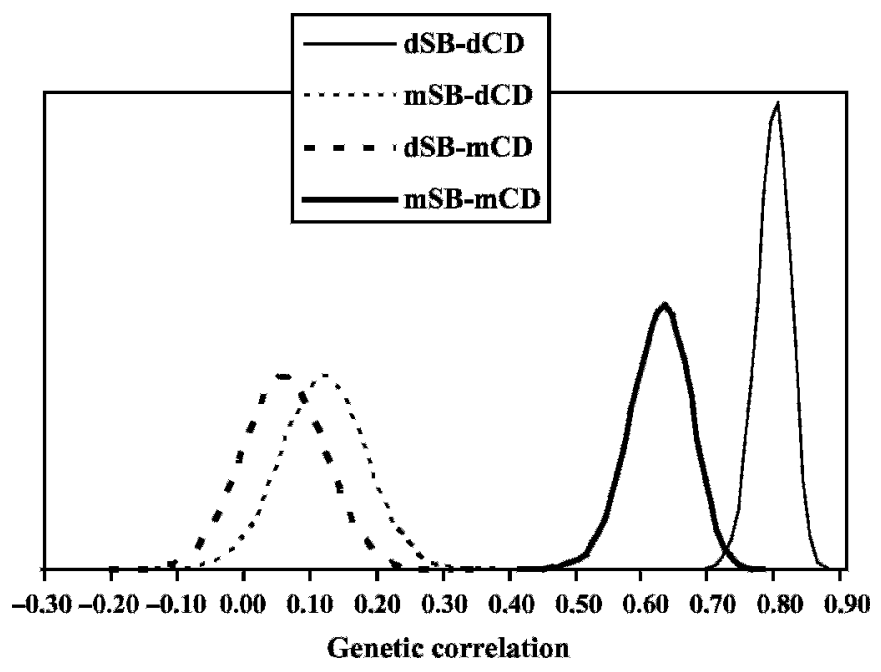

Figure 5. Posterior distributions of genetic correlation between direct (d) and maternal (m) stillbirth (SB) and calving difficulty (CD). the 2 traits was close to zero (Table 1). This suggests that herds with low SB frequency tend to have a low rate of dystocia.

\section{Genetic Change}

Figure 6 indicates that there has been little or no genetic change in stillbirth at first calving in Norwegian

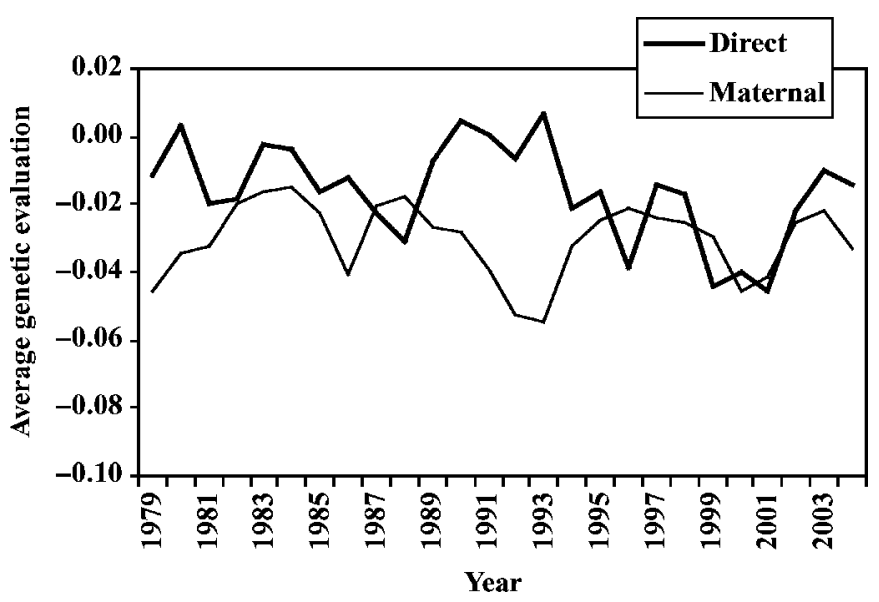

Figure 6. Genetic change for stillbirth at first calving in Norwegian Red given as average sire posterior mean by birth year of progeny (direct effect) and average genetic evaluation of sire for maternal effect by year of calving of daughters. 


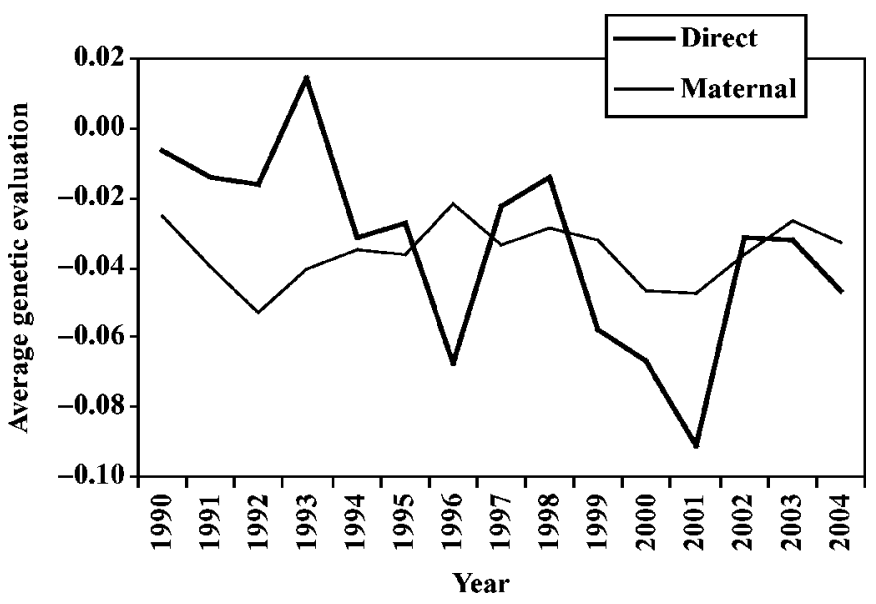

Figure 7. Genetic change for calving difficulty at first calving in Norwegian Red given as average sire posterior mean by birth year of progeny (direct effect) and average genetic evaluation of sire for maternal effect by year of calving of daughters.

Red, for either direct or maternal effects. For calving difficulty, Figure 7 indicates a slight genetic improvement for direct effect (sire of calf) and little or no genetic change for the maternal effect (sire of cow). These results are in contrast to the unfavorable genetic trends for SB and CD found for first-calving Holsteins. Hansen et al. (2004c) reported unfavorable genetic trends for both direct and maternal effects of stillbirth and for direct effect of CD in the Danish Holstein population, whereas no trend was observed for maternal calving difficulty. In the United States there has been an increasing trend for mean service sire PTA for percentage of difficult calvings for first-calf daughters over the last 10 yr (Van Tassel et al., 2003). Meyer et al. (2001b) reported an annual genetic change of -0.04 and -0.02 for direct and maternal effects for perinatal survival, which is the opposite of stillbirth, for US Holstein bulls born from 1984 to 1994.

The levels of stillbirth and calving difficulty are very low in the Norwegian Red population; therefore, there is not much scope for genetic improvement. However, because of their economic and animal welfare relevance, it is important to keep these traits in the total merit index used for selection of Norwegian Red sires, as well as to avoid using sires that are extremely bad for these traits. In populations with higher and increasing frequency of SB and $\mathrm{CD}$, more weight should be placed on calving traits to avoid further deterioration.

\section{CONCLUSIONS}

Levels of SB and CD in Norwegian Red are low, with an overall mean (all parities) stillbirth rate of about $2 \%$, and $95 \%$ of the cows reported to have an easy calv- ing. Direct and maternal heritabilities for SB and CD were between 0.07 and 0.13 . Strong genetic correlations were found between direct SB and direct CD (0.79) and between maternal SB and maternal CD (0.62). All genetic correlations between direct and maternal effects, within or between traits, were close to zero, implying that for these traits, bulls should be evaluated both as sire of the calf (direct effect) and sire of the cow (maternal effect). No genetic change for SB and a slight genetic improvement for CD were found in the Norwegian Red population.

\section{ACKNOWLEDGMENTS}

Access to the data was given by the Norwegian Dairy Herd Recording System in agreement number 004.2005. This work is part of project no 167893/I10 ("Avl for friskere kyr") financed by the Research Council of Norway. Support was also received from the Babcock Institute for International Dairy Research and Development, University of Wisconsin, Madison, and a BILAT project from the Research Council of Norway.

\section{REFERENCES}

Albert, J. H., and S. Chib. 1997. Bayesian methods for cumulative, sequential and two-step ordinal data regression models. Technical Report. Department of Mathematics and Statistics, Bowling Green State University, Bowling Green, OH.

Chang, Y. M., I. M. Andersen-Ranberg, B. Heringstad, D. Gianola, and G. Klemetsdal. 2006. Bivariate analysis of number of services to conception and days open in Norwegian Red using a censored threshold-linear model. J. Dairy Sci. 89:772-778.

Geno. 2006. Net merit index. http://www.geno.no. Accessed October 20, 2006.

Gevrekçi, Y., Y. M. Chang, K. Kızılkaya, D. Gianola, K. A. Weigel, and Y. Akbaş. 2006. Bayesian inference for calving ease and stillbirth in Holsteins using a bivariate threshold sire-maternal grandsire model. Proc. 8th WCGALP, Belo Horizonte, Brazil. CDROM Commun. no. 01-26.

Gianola, D., and J. L. Foulley. 1983. Sire evaluation for ordered categorical data with a threshold model. Genet. Sel. Evol. 15:201-224.

Hansen, M., M. S. Lund, J. Pedersen, and L. G. Christensen. 2004a. Gestation length in Danish Holsteins has weak genetic associations with stillbirth, calving difficulty, and calf size. Livest. Prod. Sci. 91:23-33.

Hansen, M., M. S. Lund, J. Pedersen, and L. G. Christensen. 2004b. Genetic parameters for stillbirth in Danish Holstein cows using a Bayesian threshold model. J. Dairy Sci. 87:706-716.

Hansen, M., I. Misztal, M. S. Lund, J. Pedersen, and L. G. Christensen. 2004c. Undesired phenotypic and genetic trend for stillbirth in Danish Holsteins. J. Dairy Sci. 87:1477-1486.

Heins, B. J., L. B. Hansen, and A. J. Seykora. 2006. Calving difficulty and stillbirths of pure Holsteins versus crossbreds of Holstein with Normande, Montbeliarde, and Scandinavian Red. J. Dairy Sci. 89:2805-2810.

Jamrozik, J., J. Fatehi, G. J. Kistemaker, and L. R. Schaeffer. 2005. Estimates of genetic parameters for Canadian Holstein female reproduction traits. J. Dairy Sci. 88:2199-2208.

Luo, M. F., P. J. Boettcher, J. C. M. Dekkers, and L. R. Schaeffer. 1999. Bayesian analysis for estimation of genetic parameters of calving ease and stillbirth for Canadian Holsteins. J. Dairy Sci. 82:1848-1858. 
Luo, M. F., P. J. Boettcher, L. R. Schaeffer, and J. C. M. Dekkers. 2002. Estimation of genetic parameters of calving ease in first and second parities of Canadian Holsteins using Bayesian methods. Livest. Prod. Sci. 74:175-184.

Meyer, C. L., P. J. Berger, K. J. Koehler, J. R. Thompson, and C. G. Sattler. 2001a. Phenotypic trends in incidence of stillbirth for Holsteins in the United States. J. Dairy Sci. 84:515-523.

Meyer, C. L., P. J. Berger, J. R. Thompson, and C. G. Sattler. 2001b. Genetic evaluation of Holstein sires and maternal grandsires in the United States for perinatal survival. J. Dairy Sci. 84:12461254.

Philipsson, J., L. Steinbock, and K. Johansson. 2006. Differences in genetic variation of calving traits in Swedish Holstein and Swedish Red cattle. Proc. 8th WCGALP, Belo Horizonte, Brazil. CDROM Commun. no. 01-24.
Raftery, A. L., and S. Lewis. 1992. How many iterations in the Gibbs sampler? Pages 763-774 in Bayesian Statistics 4. J. M Bernando, J. O. Berger, A. P. Dawid, and A. F. M. Smith, ed. Oxford University Press, Oxford, UK.

Sorensen, D., and D. Gianola. 2002. Chapters 10-12 in Likelihood, Bayesian, and MCMC methods in quantitative genetics. SpringerVerlag, New York, NY.

Steinbock, L., A. Näsholm, B. Berglund, K. Johansson, and J. Philipsson. 2003. Genetic effects on stillbirth and calving difficulty in Swedish Holsteins at first and second calving. J. Dairy Sci. 86:2228-2235.

Van Tassel, C. P., G. R. Wiggans, and I. Misztal. 2003. Implementation of a sire-maternal grandsire model for evaluation of calving ease in the United States. J. Dairy Sci. 86:3366-3373.

Wiggans, G. R., I. Misztal, and C. P. Van Tassell. 2003. Calving ease (co)variance components for a sire-maternal grandsire threshold model. J. Dairy Sci. 86:1845-1848. 